Linguistique, littérature, didactique

191-192 | 2021

Théâtre contemporain : Écritures et Représentations

\title{
La ponctuation dans les textes dramatiques contemporains
}

Punctuation in contemporary dramatic texts

\section{Michel Favriaud}

\section{(2) OpenEdition \\ 1 Journals}

\section{Édition électronique}

URL : https://journals.openedition.org/pratiques/10422

DOI : 10.4000/pratiques. 10422

ISSN : 2425-2042

Éditeur

Centre de recherche sur les médiations (CREM)

\section{Référence électronique}

Michel Favriaud, "La ponctuation dans les textes dramatiques contemporains », Pratiques [En ligne],

191-192 | 2021, mis en ligne le 15 décembre 2021, consulté le 03 janvier 2022. URL : http://

journals.openedition.org/pratiques/10422 ; DOI : https://doi.org/10.4000/pratiques.10422

Ce document a été généré automatiquement le 3 janvier 2022.

(c) Tous droits réservés 


\title{
La ponctuation dans les textes dramatiques contemporains
}

Punctuation in contemporary dramatic texts

\author{
Michel Favriaud
}

\section{Introduction}

1 La ponctuation ne se réduit pas aux signes de ponctuation. Dans le plurisystème ponctuationnel (Favriaud 2014), nous posions trois tiroirs, noir, blanc, gris (ou graphique) et un éventuel tiroir phonique (accentuel). Les tiroirs blanc et gris amènent la question de la scénographie visuelle du texte, voire celle de l'extension de la ponctuation: quelle continuité entre ponctuation et autres phénomènes de segmentation, liaison, hiérarchisation, accentuation, rythme? Il semble bien que la ponctuation soit devenue dans le théâtre contemporain un phénomène massif et protéiforme. Parallèlement le théâtre n'a cessé d'évoluer, accordant une place de plus en plus déterminante aux didascalies, objet premier de notre étude.

2 L'hypothétique ponctuation phonique, faible de n'avoir pas de répondant visuel systématique (Favriaud, 2014), a l'intérêt heuristique de réinterroger le rapport entre écrit et oral, nul selon les autonomistes de la ponctuation, comme J. Anis et G. Nunberg; persistant quoique inégal selon les phonocentristes, même modérés comme N. Catach (Anis, 2004, pour synthèse). Or le théâtre, dans une de ses séquences, la dialogale, se veut une (re)présentation et une présentification écrite de l'oral. On peut donc se demander quels moyens il se donne: assurément la ponctuation, mais aussi les didascalies dont l'une des fonctions est de souligner (ou de prescrire ?) les éléments vocaux, posturaux et contextuels de la conversation orale non notés par la ponctuation. 
3 Partant du principe que les ponctuants ont une valeur générale en langue, sans homonymie, on peut faire les quatre hypothèses suivantes reliées au théâtre contemporain :

1. Les ponctuants de la séquence dialogale et de la séquence didascalique se chargeraient de valeurs concordantes avec les valeurs générales du système ponctuationnel ;

2. Les ponctuants adopteraient en outre des emplois et valeurs caractéristiques du genre (théâtral) et de la séquence (conversationnelle ou didascalique).

3. Les ponctuants répondraient aussi des auteurs et des œuvres, voire des époques et des publics ciblés ; il y aurait ainsi une pragmatique et une poétique de la ponctuation ;

4. Les didascalies auraient un double rôle: d'adjuvant informationnel à la ponctuation de la séquence conversationnelle, mais aussi de contraste, comme séquence écrite n'ayant pas l'obligation, elle, de mimer l'oral.

4 La question sous-jacente est bien le régime du texte théâtral, scindé en deux séquences majeures : est-il un texte oral transcrit et annoté, ou un texte écrit hybride?

5 De surcroit, les différents tiroirs ponctuationnels engagent des unités discursives caractéristiques (Favriaud, 2014). Nous essaierons de démontrer qu'au théâtre la phrase, conventionnellement typique de l'écrit (Benveniste, 1966, 1974), et la période, typique de l'oral (Lacheret et Victorri, 2002) non seulement coexistent, mais aussi qu'elles cohabiteraient avec d'autres unités discursives. La ponctuation révélerait ainsi une (première) scénographie du texte, comprise comme l'architecturation d'unités discursives multiples, emboitées ou concurrentes. Aussi notre analyse tournera autour du couple unités discursives-ponctuants.

6 La question finale serait de savoir si cette scénographie discursivo-ponctuationnelle complexe sera prescrite par la ponctuation, aidée par les didascalies, ou sera un lieu de négociation métalinguistique et poïétique entre auteur et lecteur.

7 Pour ce faire, nous alimenterons notre corpus francophone de la fin du XX et du début $\mathrm{du} \mathrm{XXI}^{\mathrm{e}}$ siècle, à trois sources : les pièces européennes, les pièces extra-européennes (dont le français pourrait être traversé par une autre langue, maternelle) et quelques pièces de littérature de jeunesse.

\section{Le point sur le plurisystème ponctuationnel}

Résumons notre plurisystème (Favriaud, 2014).

\subsection{La ponctuation}

\subsubsection{La ponctuation noire :}

On peut distinguer les ponctuants de démarcation externe et ceux de construction interne: la ponctuation des limites discursives et la construction médiane (de la phrase, par exemple). Tous ces signes ont des valeurs proches, celles d'actualisation du discours, de segmentation et de liaison, mais avec des intensités distinctes (Favriaud, 2011). Certains signes médians seront dits forts, comme le deux-points et le tiret, plus sécants et plus accentués, certains plus faibles, comme le point-virgule et surtout la virgule. La ponctuation de fin d'unité discursive prend une valeur modale d'intensité 
variable: le point aura une valeur modale générale zéro, les points d'exclamation, d'interrogation et de suspension, une valeur modale suspensive à préciser.

On a l'habitude de penser les signes de ponctuation noire dans le cadre de la phrase, mais ils peuvent s'analyser dans des cadres discursifs plus larges où ils ont un double rôle, final ou médian. Ainsi le point peut terminer un paragraphe ou une réplique, mais devenir médian dans le corps de ceux-ci. Cette "médianisation" généralisée des ponctuants (Favriaud, 2014) et cette valeur différente selon le cadre discursif envisagé seront prises en compte.

\subsubsection{La ponctuation blanche :}

11 Le blanc devient tiroir ponctuationnel à part entière dans la mesure où il possède lui aussi ses marques, ses unités discursives de prédilection et sa syntaxe. La ponctuation blanche n'a que des marques différentielles («en négatif» dit N. Catach) qui se caractérisent par l'épaisseur du blanc et par le nombre de faces blanchies de tel texte ou partie de texte. Plus un segment est blanchi, plus il acquiert d'importance, d'autonomie et d'alinéarité. Les unités discursives actualisées par la blanche sont le texte, la scène, la réplique, le verset, le vers, et d'une certaine manière, le titre.

Quand la blanche redouble la noire, elle donne à celle-ci sa dimension et sa portée. Ainsi la distinction entre point final de mot (abrégé), de phrase, de période, de réplique, de scène, d'acte, de pièce dépendra en grande partie de la taille du blanc de droite. On distinguera deux emplois majeurs de la ponctuation blanche: un emploi complémentaire de la noire, ce qui pose la question de l'ancillarité de l'un par rapport à l'autre, et un emploi autosuffisant (D.-G. Gabily dans Chimères clôt chaque réplique uniquement par un blanc avec alinéa). Dans les deux cas, la syntaxe diffère; l'unité blanchie autonome est réglée en grande partie par la parataxe; elle provoque la sortie au moins partielle de la linéarité discursive, comme l'illustre le cas du titre, avec majuscule initiale mais sans point final, qui fait phrase, souvent nominale, mais pas vraiment première phrase du texte ; plutôt hyperphrase jouant avec tout le texte.

La ponctuation blanche dans le théâtre contemporain prosé (rarement versifié tout du long) participe donc fortement à la construction du texte et à la mise au jour de son plan, de ses échelons discursifs distincts et d'une potentialité alinéaire, permettant, en lecture, et peut-être en mise en scène contemporaine, des parcours multiples et créatifs.

\subsubsection{La ponctuation grise :}

Ce tiroir concerne les transformations graphiques de police, de taille, de couleur qui actualisent une hétérogénéité discursive. Les éléments ainsi grisés ont un quadruple emploi :

1. ordinaire, comme élément pris dans la concaténation discursive ;

1. extraordinaire comme émanant d'une source hétérogène, provoquant écart, mise en relief et en relation, et donc effets de sens ;

2. distinctif de deux séquences principales, actualisant presque toujours, par l'italique, la didascalie: sorte de note, de bas de page, de haut de page, en chapeau sous le titre, ou d'accompagnement cotextuel immédiat. 
3. constitutif d'un continu didascalique potentiel - notamment dans certains cas de prolifération contemporaine des didascalies en taille et en nombre - dont les différents morceaux pourront être aussi considérés en continuité et relation. En français, à l'accent ordinaire de clôture syntagmatique, s'ajoutent les accents métriques et prosodiques (Dessons et Meschonnic, 1998), qui tous provoquent de nouvelles concaténations sémantiques. On partira du principe que tous les ponctuants accentuent au moins la syllabe d'amont et généralement plus faiblement la syllabe d'aval, mais que, inversement, tous les accents ne bénéficient pas d'un ponctuant ordinaire, les moins marqués notamment. Le questionnement de J. Damourette sur la "pausette", indexée par une virgule ou non, nous a fait poser l'hypothèse d'un ponctuant faible d'un nouveau type, phonique à visibilité zéro (Favriaud, 2011).

Donc, autant que d'un quatrième tiroir, il s'agirait plutôt d'une extension possible de la ponctuation aux accents et au ponctuant à visibilité zéro, et de l'agrément d'un principe phono-accentuel caractéristique de tous les ponctuants que les neurosciences semblent accréditer en montrant la stimulation neuronale en lecture d'écrit de zones considérées jadis comme spécifiquement dédiées à l'oral (Kolinski et al., 2014). Ce qui rendrait la frontière entre écrit et oral poreuse, par la ponctuation notamment.

\section{Le point sur les unités discursives actualisées par la ponctuation}

\subsection{Une théorie des unités discursives indexée par la ponctuation : l'exemple de la phrase:}

Dans les études linguistiques standard, on prend peu en compte la ponctuation, considérée comme ancillaire ou fantasque. Ainsi A. Berrendonner et M.-J. Béguelin refusent d'accorder le moindre rôle à la ponctuation noire des limites, la seule ponctuation qu'ils envisagent malheureusement, et donc à la phrase (Berrendonner, 2002, 2021 ; Béguelin, 2002). Au fameux propos conclusif d'A. Berrendonner : « Il n'y a pas de phrases, ni dans la langue, ni dans le discours " (2002, p. 34), M.-J. Béguelin ajoute :

Une théorie des unités est-elle vouée à calquer les pratiques (ortho)graphiques, ou au contraire doit elle s'en abstraire (ibid., p 88-89) ?

Autrement dit, elle se propose de passer par-dessus les signes positifs les plus saillants du discours écrit que sont les ponctuants, et pour ces raisons :

Qu'en est-il comparativement, de la ponctuation, ce système de renfort de l'écriture apparu secondairement, et dont se passent non seulement les scripteurs débutants, mais aussi beaucoup de poètes ou d'écrivains? [...] À un même ponctuant 
correspondent habituellement plusieurs valeurs, parfois simultanément, parfois en

fonction du contexte » (ibid., p. 91). permettra de mettre au jour phrase, paragraphe, chapitre, et, dans une moindre mesure, période, laquelle sera distribuée en deux sous-catégories: intraphrastique, segmentée par au moins un médian moyen ou fort (point-virgule; deux-points, tiret entre autres - sans avoir recours à la notion bâtarde de «sous-phrase » de M. Wilmet (1997, p. 436), et interphrastique (phrases distinctes mais reliées par une rection syntaxique en une unité supérieure, comme défendu par M. Charolles [1988, p. 7]). La co-occurrence des phrase et période permet d'élaborer un modèle explicatif plus puissant que la pluriséculaire négation de l'une par l'autre. On retrouverait probablement à l'oral cette même dyarchie, mais cette fois - c'est notre hypothèse avec une dominance de la période sur la phrase. La réplique théâtrale sera, au même titre que le paragraphe, une unité noire supérieure.

La ponctuation blanche permettra de mettre au jour le segment blanchi (et donc le vers et le verset) mais aussi, au plan macrotextuel, le texte et ses parties fortement blanchies, acte, scène et leurs succédanés. Dans ces cas de cohabitation de ponctuation noire finale et de blanc élargi, il semble que la blanche domine la noire, au point que la noire puisse disparaitre (Gabily, 1994). La structuration du vers sera plus ou moins concurrente de celle de la phrase ou de la clause, sans emboitement hiérarchique strictement coïncidant (eu égard aux phénomènes d'enjambement). linéarité, séquences ou segments courts ; ces derniers pourront se recombiner dans des configurations plus parataxiques, nos « unités fluctuantes » (Favriaud, 2014), comme le feront aussi les éléments accentués phoniquement, par prosodie sérielle.

L'architecturation du texte, éclairée par la ponctuation étendue, résultera des complémentarité, intrication et éventuelle concurrence de ces unités discursives, sources de polysémie. 


\section{Les didascalies : types, places et fonctions} avec une ponctuation noire finale de segment et une ouverture vers le discours direct

- point simple et retour à la ligne (L'Amour de Phèdre de S. Kane)

- point simple (Albertine; Journée de noces chez les Cromagnons, de W. Mouawad, et tous les textes d'Actes Sud-Papiers) et majuscule d'entame de la phrase suivant immédiatement, ce qui donne au nom propre les attributs complets de la phrase nominale; seule la capitalisation de ce nom signale l'hétérogénéité dialogique.

- point final suivi d'un tiret d'ouverture : B.-M. Koltès, Quai Ouest, J.-L. Lagarce, Juste la fin du monde; cadratin chez D. Lemahieu, Théâtre I. Le tiret a pour valeur la rupture énonciative, notamment dans le changement d'interlocuteur et l'accentuation phonique d'amont et d'aval qui en résulte. On sait, au moins depuis P. Larthomas, qu'au théâtre la forte attaque de 
réplique doit capter l'attention, créer un événement et permettre la compréhension immédiate d'un énoncé non enchainé.

- deux-points, à la suite de la mention du nom du locuteur en gras (Musika) ; le deux-points ne crée pas le changement de phrase mais introduit une complexité énonciative, une rupture et une ouverture, à peu près équivalente à celle du tiret, mais souvent plus dynamique. Médian, il ne peut être cumulé avec un signe noir final, ce qui exclut la notion de phrase nominale de départ, mais pas la notion de période intraphrastique.

Ainsi prend-on conscience que les solutions ponctuationnelles sont variées, sans nuance de sens sensible : finalement les signes s'équivalent pour indexer la mise en relief, la rupture énonciative et le passage à niveau vers une nouvelle énonciation, bénéficiant d'un accent d'attaque. Au rôle prévisible de désambiguisation locutoire, il s'en est ajouté quatre autres : 1) le repérage des limites de la réplique comme unité majeure du discours théâtral ; 2) le changement de régime discursif, de l'écrit vers l'oralité et la théâtralité, souligné par la forte concentration ponctuationnelle à la charnière entre didascalie attributive et discours direct ; 3) la contre-accentuation forte de la première syllabe et du premier mot du discours conversationnel, pour ainsi dire projetés par le personnage qui doit capter immédiatement l'attention ; 4) le rythme cognitif et affectif, causé par la récurrence et l'alternance de ces étiquettes proéminentes, fondé à la fois sur le retour du même, l'alternance et l'irrégularité significative tant des prises de parole que de leur longueur. Les ponctuations blanche et grise très saillantes de ces étiquettes nominales, couplées avec les titres et ponctuations de début et de fin de scène, permettent au lecteur de préformer mentalement la scène entrevue, et de s'y accorder rythmiquement et émotionnellement. Ainsi, le lecteur de théâtre peut avoir, pour une fois, une longueur d'avance sur le spectateur.

\subsection{La didascalie informative et rythmique}

33 Au plan de la ponctuation, la didascalie stricto sensu (hors désignation des locuteurs) apparait presque toujours en italique (sauf chez V. Novarina, Le Drame, avec seule ponctuation blanche paragraphique, peu distinctive), marquant son hétérogénéité énonciative et son ancillarité, comme appendice du "vrai » texte théâtral qui serait sa séquence conversationnelle. Cette ancillarité peut être soulignée par les ponctuants suivants : parenthèses du texte didascalique et, quelquefois, diminution de taille de caractères (J.-M. Dalpé, Le Chien), ce qui ouvre la possibilité de plusieurs types de didascalie co-présents, correspondant peut-être à des voix ou à des fonctions pragmatiques distinctes. Le cas plus radical de V. Novarina (Le Drame) tend vers la nondistinction des séquences didascaliques et conversationnelles, ce qui ne manque pas d'avoir des conséquences dramaturgiques et scéniques.

On distinguera les didascalies courtes au fil des répliques, les didascalies intermédiaires entre répliques ou entre sous-parties de scènes, et les didascalies de début et de fin de scène.

\subsubsection{Deux types de didascalies courtes au fil de la réplique :}

Dans C. Zambon (Sissi, p. 51) :

À Sissi : Tiens. Regarde. (Il lui donne l'oiseau qui est tout mouillé, plein de boue. Elle le remercie.) Faut qu'il sèche. Il est comme moi, il pue, il est plein de boue et tout couillé, euh non (il se reprend), tout mouillé, mouillé, mouillé, mouillé. 
Il la regarde, inquiet. Elle ne se moque pas de lui. Ça le rassure.

Je ne peux pas te donner Méli, je ne peux pas. C'est Méli, ça ne se donne pas.

Lionel désigne Méli.

Il est né en même temps que moi, tu comprends? on relève trois traitements ponctuationnels de ces didascalies. La première occurrence, courte (« ̀̀ Sissi»), fonctionne un peu comme l'étiquette d'attribution locutoire, sauf qu'il s'agit ici d'interlocution et d'interlocuteur, avec: retrait de marge, majuscule d'entame, deux-points ouvrant sur le discours à tenir; strictement informative. La deuxième est à l'intérieur d'un paragraphe conversationnel correspondant à une série de phrases regroupées en période interphrastique. Ici la didascalie («Il lui donne [...] ») coupe la période et loge ses deux phrases entre les parenthèses. On remarque que cette didascalie relativement longue donne des informations nouvelles, indispensables, et d'autres redondantes : «tout mouillé, plein de boue» / "Faut qu'il sèche, il est plein de boue, tout couillé, tout mouillé » (quatre occurrences de "mouillé »). Le rôle de cette didascalie n'est donc pas uniquement informatif, comme le sera «Lionel désigne Méli «, ni complètement narratif comme la troisième (chaine exhaustive des micro-actions : «Il la regarde [...] »).

dernière est autrement ponctuée en blanc et gris : blancs d'alinéa en amont et en aval qui lui permettent de s'extraire davantage de la linéarité. Cette didascalie dit beaucoup en trois courtes phrases formant période, plus que ne peut en jouer, peutêtre, l'acteur: elle est à la fois informative de l'environnement conversationnel, et narrative. On pourrait ajouter que toutes ces didascalies, sauf peut-être la première, sont nettement, en lecture au moins, rythmiques: elles découpent la réplique en moments, séparés par des pauses, des reprises toniques (accent d'attaque consonantique ou coup de glotte vocalique - selon Dessons et Meschonnic, 1998); la langue saute alors d'un régime conversationnel plus oral et enfantin, à un régime plus écrit et plus tenu d'adulte, ce qui ouvre à une autre théâtralité du langage, un dialogisme des régimes linguistiques et des niveaux de langue. On peut se demander si ces didascalies en italique ne provoquent pas elles-mêmes un effet de ponctuation suspensive, favorisant les images mentales, éventuellement le rêve du lecteur. Ce qui amène de redoutables problèmes mais aussi de belles possibilités de traitement scénique.

Il nous a été facile de distinguer visuellement les didascalies parenthétiques, dans le fil du discours, des didascalies non parenthétiques, blanchies, avec retour à la ligne, sans que les effets sémantiques de cette distinction de ponctuant soient aussi évidents.

En complément de leur apport sémantique différencié, les didascalies transversales de C. Zambon et de D. Lemahieu (Théâtre I, p. 116, 117) semblent avoir un rôle rythmique plus ou moins important, devenant en quelque sorte des macroponctuants discursifs. Leurs fonctions seraient triples : informative, complémentaire d'oralité, et poéticorythmique, dans des proportions variables. Dans les occurrences de "grognements », "feulements ", "bégaiements ", les parenthèses vont dans le sens d'une minoration des fonctions 1 et 2 ; c'est la répétition avec variation qui amène tout à la fois la fonction 3 et une majoration de l'ensemble de ces didascalies, lesquelles forment une sous-séquence didascalique homogène quasi-suprasegmentale. 


\subsubsection{Les grandes didascalies d'amorce de scène:}

de scène 23 est informative : personnages, décor et environnement sonore :

De l'autre côté du rideau. Naze et Mina, en costume de scène de la Belle et de la Bête. Au loin, de temps à autre, les échos de la représentation. Naze, Mina.

Ces quatre phrases, bien que reliées, ne sont pas toutes soumises à la même base de rection: elles forment moins une période qu'un paragraphe. Celui-ci est isolé par l'italique, blanchi au-dessus et un peu moins au-dessous, malgré l'alinéa. La numérotation de scène, le blanc à la fin de la scène 22 et entre le numéro de la scène 23 et la didascalie d'entame, s'y ajoutent pour faire un macro-ponctuant de scène.

À la scène suivante, on retrouve les mêmes éléments, mais avec une didascalie plus longue qui fait sa place à la fonction 3, rythmico-poétique (ibid., p. 109) :

Le rideau de la baraque de foire se lève. Une table est dressée. Un souper aux chandelles. Mina s'empiffre comme une boulimique, au point d'avoir parfois des haut-le-corps, ce qui ne l'empêche pas de s'empiffrer encore mieux après, comme si son corps était une bouche qui avalait et vomissait. Naze, Mina.

Des cinq phrases faisant paragraphe didascalique, l'une apparait plus longue et complexe, très redondante sémantiquement, avec répétition du verbe « s'empiffrer » et du modalisateur « comme » : " comme une boulimique », « comme si son corps » - qui amènent les accents de prosodie sérielle - et élargissement rythmique par des groupes de plus en plus longs, segmentés par une virgule: soit en nombre de syllabes hors féminines : $9 / 9$ ou 10/15/16. On peut dire que les fonctions 1 et 3 sont ici satisfaites.

À la scène suivante 25 , la didascalie stricto sensu est réduite à la fonction 1 :

Naze, seul devant le rideau.

Cependant le discours suivant tenu par Naze, toujours en italique, semble intermédiaire entre didascalie et réplique (ibid., p. 111) :

NAZE. - Et je piétine, je piétine toujours sur place, toujours sur place, je tourne en rond, je piétine comme si j'avais le vertige, mais ne pouvais pas m'arrêter, je piétine comme si j'avais mal au cœur mais ne pouvais pas m'arrêter, je piétine comme je mords ma langue, comme j'arrache ma peau [...]

C'est bien l'italique qui nous interroge, comme ponctuant habituel de didascalie, alors qu'un personnage sur scène assume son énonciation par un «je " répété. On pourra justifier l'italique ici, comme dans d'autres scènes conversationnelles, par le relatif hors-jeu ("devant le rideau») et le démarquage de La Belle et la Bête. Toutefois ce monologue présente plus d'une similitude avec la didascalie précédemment étudiée : description d'action qui n'est pas forcément jouée, répétition généralisée, comparaisons multiples avec le même modalisateur "comme", les mêmes images, passant de « haut-le corps » à « mal au cœur », et discours infini, litanique.

Nous ne dirons pas que ce monologue assumé en « je » par " Naze » est une didascalie, mais que la distinction entre didascalie à fonction rythmico-poétique et monologue répétitif, sans avancée narrative factuelle, s'est fortement estompée.

Dans D.-G. Gabily, la première scène est une immense didascalie de deux pages, qui situe bien le cadre et le premier personnage (Chimère, p. 11) :

Figure de plateau. Vent. Il y a du vent, peut-être. Premier lieu. Ainsi énoncé : [...]

Et une femme après un temps conséquent de l'ordre de la minute ou plus [...] passe

à son tour. 
Certes la fonction 1 est sollicitée, mais déjà un peu ébranlée par le modal " peut-être », séparé de l'énonciation principale par la virgule, ici accentuante. En fait, le vent va continuer à s' « énoncer » ainsi :

Vent coulis sur un petit bout du champ d'épandage du monde, à cet instant, tout au moins sur le devant, encore assez coquet, quoique peu discernable car la lumière vient à contre-jour sur le lointain où quelques portes disent, quelques chambranles de portes disent ou, si l'on veut, quelques larges trouées (cinq au minimum, sortes peut-être de portiques à l'antique dans le genre gréco-stalinien [...]

Cette période de dix lignes donne bien des informations, mais semble les réfuter et les modaliser au fur et à mesure, ce que l'on attendrait plus dans le conversationnel que dans le didascalique. Il y a un véritable suspens de la période car les éléments lancés d'abord tardent à trouver leur assise syntaxique ; c'est la période intraphrastique qui devient l'événement, non pas narratif et dramaturgique, mais poétique. Rétablissons l'énoncé ajouté entre tirets, qui prend un effet de sens parenthétique, suspensif et dispersif :

Et une femme après un temps conséquent de l'ordre de la minute ou plus - nous laisser seul avec le vent, avec l'obscurité du devant et la lumière terne d'un peutêtre jour finissant - passe à son tour.

Une autre anomalie est survenue, par le "nous » et l'infinitif : " nous laisser seul ", indiquant ordre ou conseil d'une parole dialogique assumée à la dérobée par un narrateur-poémateur-metteur en scène ${ }^{1}$ indistinct. Cette fois, c'est donc la didascalie qui est traversée par un « vent » conversationnel, aux contours flous. Un peu plus loin dans cette première scène didascalique nous aurons :

On dirait qu'elle chante. On dirait qu'elle porte un enfant. C'est une poupée. Un chiffon de corps inerte. Berçant :

«Doucement / Tire pas tout / Hop là / Mon gamin / Tête pas tout mon sein / D'un coup / Voraçou / T'arraches ta mère / Hop là / Bon sang / Bois pas tout / Comme ton ivrogne de père... »

A peine audible, évidemment, le chant. Ne pas réveiller les morts et se méfier des vivants endormis, pense-t-elle, doit-elle penser, si elle pense. [...]

La référence indéfinie $d u$ «on », la déclinaison modale du verbe "penser», la restriction d'audibilité et de plausibilité de la comptine tenue, l'adverbe modal qui brouille la vérité (" évidemment »), gomment les frontières entre réel et hypothétique, entre récit, dialogue théâtral et commentaire d'encadrement. La ponctuation noire de cette parole, à la fois comptine, berceuse et dialogue oral, segmente les énoncés à coup de barres obliques, qui contestent la linéarité hypotaxique et la complétude du discours, suggérant le monde hypothétique plus rêveur, plus épars de la poésie.

La didascalie en italique a rempli partiellement les trois fonctions, mais en a jouté au moins deux autres qui ont partie liée avec la ponctuation : elle a pu incorporer, ce qui semblait contradictoire avec sa définition séquentielle, du conversationnel, mais à un autre niveau, avec un autre statut : d'abord, celui de la sous-conversation non adressée ou multi-adressée, plus poétique que réellement informative; ensuite elle a servi d'entame de scène voire de pièce, devenant ainsi un macro-ponctuant des grandes unités discursives théâtrales : pièce et scène. 


\subsubsection{Les didascalies de clôture de macro-unités}

Il arrive souvent que les didascalies clôturent une scène, voire la pièce entière, mais dans ce cas-là, elles sont rarement longues. Une phrase dans M. Tremblay, Albertine ( $\mathrm{p}$. 58) :

LES CINQ ALBERTINE (comme si elles avaient un contact physique). Haaa...

La lune, solitaire et rouge sang, se lève. séparation ponctuationnelle entre sujet et verbe par l'apposition qui suspend le rapport sujet/verbe, neutralise le cliché («La lune [...] se lève ») et emphatise poétiquement le moment final. L'apposition, outre son apport sémantique, apporte une dimension au ponctuant faible entre sujet et verbe, habituellement constitué d'une " pausette », avec ponctuant phonique à visibilité zéro (Favriaud, 2010). la lune-sang configurent un macro-ponctuant final de pièce, à la fois modeste, peu détaché et moyennement blanchi - en pianissimo final.

-M.Dalpé, Le Chien (p. 97), la didascalie prolonge aussi la dernière réplique, élucide l'environnement langagier, le geste d'accompagnement et la portée des paroles de Jay, le fils revenu pour venger sa sœur enceinte, probablement du père :

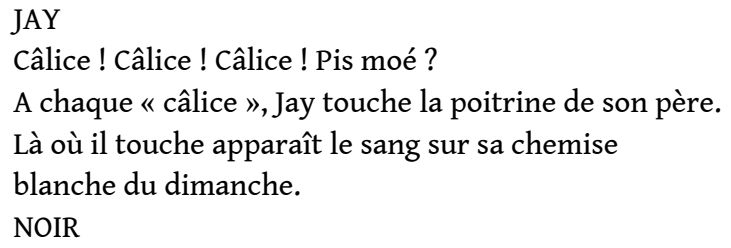

vons là deux ponctuants gris distincts, minuscules/majuscules, caractères droits/italique visualisant une partie du feuilletage de la sur-énonciation théâtrale, en plus des points d'exclamation et de la répétition de "câlice ", allongée et suraccentuée dès la première syllabe par l'accent circonflexe non canonique, marqueur d'oralité idiolectale ${ }^{2}$. La double didascalie clôt la pièce, d'abord par la triple ligne en italique avec des retours blanchis à la ligne qui évoquent la poésie versifiée et mettent en relation les mots détachés par le blanc comme: "père ", "chemise/blanche", "dimanche". Ensuite par le mot «NOIR» qui figure, par le lexique et les ponctuants gris et blanc, la clôture du texte (ou augmente la dimension du point final précédent). On remarquera que ce «NOIR » bien qu'en police de caractère différente, n'est pas blanchi par un large espace supérieur, ce qui le met en rapport plus étroit avec "dimanche » («noir »), avec " chemise/blanche" et possiblement avec "câlice " même. "NOIR " est en même temps dans la linéarité et hors d'elle. Dans le texte et sur le plateau: devenant alors ponctuant théâtral bicodique.

Ainsi, didascalie et ponctuants entrecroisés figurent le «supplément 》 (rousseauiste ?) ineffable du discours. La didascalie devient un ponctuant final de texte, à fonction expressive forte, polysémique - le contraire des émoticones et smileys contemporains, plus simplistes, moins trempés d'imaginaire.

\subsubsection{Les didascalies comme ponctuation médiane de macro-unités}

Il arrive même par exception que les didascalies deviennent la séquence majeure, numérotée comme scène à part entière, englobant éventuellement des dialogues, ce qui 
mine le dispositif théâtral traditionnel. Est-on dans un Nouveau Théâtre (vs Nouveau Roman) ou fait-on au contraire retour vers le théâtre grec ou baroque ? Qu'est-ce que la ponctuation étendue nous en montre?

Dans Musika, d'A. Tarnagda, intervient justement un "coryphée » tout au long de la pièce divisée en dix scènes, et plus particulièrement dans les "prologue » et " épilogue ». La scène 7 court sur une demi-page, toute en italique, sauf à l'extrême fin du dernier alinéa (p. 28) :

Dans cette majesté de la nuit, on entend la voix de Wamba susurrer : «C'est fini ma fille.

C'est fini... » didascalique : la scène 3 est globalement en italique, mais les dialogues englobés - et les étiquettes attributives en petites capitales suivies d'un point - le sont en caractère droit. La ponctuation quasi inversée nous indique bien qu'il s'agit d'une contre-scène. De même, il se passe des événements, avec des personnages nouveaux, «la femme ", "le mari », mais pas vraiment identifiés, au degré de réalité fortement modalisé et atténué (Chimère, p. 16) :

Puis tout cela se perd, cesse. Une dernière pierre tombe de ce mur qui se serait effondré et c'est le grand jour à nouveau ou ce qui y ressemblerait le plus. Ils ont peut-être joué tous les deux, qui sait, le jeu de rôles - en ce cas, le Servant avait à se débrouiller avec la mère et toute la figuration : le Maître aime à singer les pères méfiants.

Ce passage didascalique est à la fois informatif, narratif et explicatif, mais avec une forte dose de modalité d'incertitude et d'illusion. Dans le dernier paragraphe, on ne sait si le Maître est encore dans son jeu de rôle - celui du père s'offusquant de l'attitude racoleuse de sa fille dans une soirée dansante - ou dans celui, très archétypal au théâtre, du maitre et du serviteur (ibid., p. 16-17) :

Le Maître avait levé le bras, secoué la main au bout de ce bras, comme un adieu sénile, ou comme s'il avait voulu - mais calmement - que cette main s'arrachât de son bras, dans la stupéfaction que cette main ne s'arrache pas encore tout à fait de son bras. Puis l'épaule idem, mais moins longtemps. Puis le Maître a redescendu son bras. Maintenant c'est terminé. Plus tard, il parlerait à nouveau :

MAÎTRE. L'histoire. L'histoire commence, commençait toujours ainsi. Il y avait toujours quelqu'un qui me regardait [...] 

allons étudier maintenant.

\section{La didascalie, simple pont ponctuationnel entre l'écrit et l'oral ?}

72 Nous défendons la conception phonocentriste modérée selon laquelle la ponctuation est liée à l'oralités, tout en admettant que la ponctuation ne fournit pas tous les traits d'oralité, ni en quantité ni en précision. Dans la séquence conversationnelle, le dramaturge essaie de donner l'illusion de l'oral, tout en le transcrivant par écrit; la ponctuation donne alors bien des informations d'oralité sur la segmentation du discours, son architecturation et ses modalités de base ; on vérifiera aisément que les ponctuants de modalité suspensive (interrogative, exclamative et suspensive proprement dite) sont, au moins en quantité, caractéristiques de l'agôn conversationnel du théatre. Mais le plus souvent les ponctuants ne suffisent pas (Petitjean, 2012). Le rôle de la didascalie serait de compléter le façonnage mimétique de l'oral (notre fonction 2), 
de réintroduire les autres langages cooccurrents ainsi que l'environnement matériel et situationnel de la conversation (notre fonction 1 ). Sont-ce bien ses uniques fonctions?

\subsection{La didascalie en complément du langage oral : volume, débit, timbre, intonation}

La ponctuation rend compte médiocrement des modalités et de l'intonation, et fait souvent l'impasse sur le volume, le débit, le timbre, et toute la pluricodicité conversationnelle (gestes, mouvements, attitudes, etc. - voir Bottineau, 2013). Notre hypothèse est que les paramètres de la voix sont en partie explicités par l'auteur dans les didascalies, en partie déductibles du discours oral lui-même, selon la posture du dramaturge.

Dans C.Zambon, les traits d'oralité seront assez facilement inférés. Voici le relevé exhaustif des parties de didascalies éclairant notre propos (Sissi, p. 11-25):

11 : C'est Méli. Il lui parle [...] Parfois il semble l'écouter. [...] Lionel chante [...]

12 : Il recommence à chanter, en hésitant, comme s'il inventait les paroles au fur et à mesure

[...]

Il crie de joie :

13-14: (il se reprend) X3

Il s'énerve un peu :

15 : linterrompant

17 : (il se reprend) $\mathrm{X} 2$

Il crie en direction de sa mère.

19 : Elle bredouille un peu :

21 : Il chantonne à Méli en se coiffant comme l'a décrit sa mère :

22-23: Il appelle :

Il semble perplexe.

Il se calme :

(Il imite la voix de Mme Defavet :)

(il se reprend) $\mathrm{X} 2$ ponctuation n'apportera jamais : "il imite la voix de Mme Defavet », soit au contraire une information redondante comme "l'interrompant", "se reprend", "bredouille», que la ponctuation et/ou le lexique explicitaient déjà.

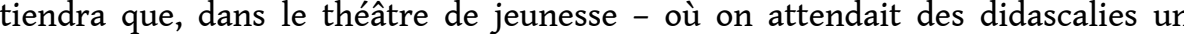
apport exclusif de précisions communicationnelles (fonction orale 1) et vocales (fonction orale 2) vicariantes de la séquence conversationnelle - les trois fonctions jouent " en même temps ", et que la troisième, rythmique, vient toujours s'ajouter aux deux autres.

81 J.-M. Dalpé fait un peu exception pour le volume, quand de la ponctuation grise il utilise ce qu'on appellerait, en bande dessinée, la graphiation (Le Chien, p. 69) : 
J'me suis pas tenu souvent avec des femmes enceintes. Entre les chantiers de construction pis les chambres de motel, c'est pas ça que tu rencontres... (Vivement :) TABERNAC D'OSTIE DE CÂLICE !!! le point d'exclamation triplé, les points de suspension quasi introductifs, pour donner $\mathrm{du}$ volume visuel et sonore. Mais, de surcroit, la mention didascalique « vivement » (le vivace de la musique), suivie du deux-points ouvrant, règle explicitement, sinon précisément, le débit, sinon le volume.

\section{8) : «(Elle rit.)» probablement parce que le discours oral les induit (ibid.) :}

Tu es toujours là, à croire, je ne sais pas, n'ai jamais compris, toujours là, toujours été là à croire qu'on te veut du mal, te faire tort, qu'on cherche à te nuire. Taciturne ce n'est pas un défaut, c'est un défaut? Paul? Ce n'est pas un défaut, je connais des tas de gens charmants tout à fait taciturnes, et pourtant fréquentables... Oh, et puis, qu'est-ce que cela fait ?...

On notera d'abord que les répliques des personnages sont longues, ici de deux tiers de page, ce qui donne à ceux-ci (et au lecteur) le temps de construire la situation, la personnalité et le type de discours. La ponctuation, la segmentation, la construction syntaxique des phrases et de l'interphrase, la modalité, souvent interrogative et suspensive, les répétitions permettent d'induire - mais avec des marges de liberté accrues - le débit, le mode de penser et de communiquer, tout en suspendant volume, timbre et intonation.

D.-G. Gabily, lui, mine la frontière entre didascalie et dialogue, notamment par la ponctuation, comme dans cette scène 9 , fortement didascalique (Chimères, p. 29) :

Maître: il se retourne, il la regarde, ils se rapproche. Un mouvement de main vers elle agenouillée, vers ses cheveux. Il ne la touche pas. Il ne les touche pas. Il est penché au-dessus du poupon. Ils font la crèche ou quelque chose qui y ressemblerait sans les agneaux, sans les bergers et sans les anges. Ça ne dure pas trop, évidemment. Il dit:

MAÎTRE. Ça ne va pas, vraiment

Il repousse légèrement du pied le poupon. La Gamine dit :

LA GAMINE. Qu'est-ce que tu fabriques, mon Joseph. [...]

Ici, quasi-absence de mention, directe ou indirecte, de la sphère sonore. On pourrait se demander pourtant si le rythme syncopé des clauses et des phrases des didascalies ne construit pas, très indirectement, une atmosphère vocale. L'absence de clôture ponctuationnelle des répliques entraine une absence des points d'exclamation et d'interrogation attendus.

Le plus surprenant est cette étiquette attributive en tête de didascalie (Maître), typique $\mathrm{du}$ lancement conversationnel, qui ne se distingue de l'occurrence prédialogale suivante que par la police de caractères (MAîTRE). Les phrases, majoritairement très courtes, proposent une suite diégétique de micro-gestes et actions. La fin inattendue de cette didascalie nous projette même dans l'univers du récit dialogué par: "Il dit:", comme plus loin: "La Gamine dit:». Les deux séquences, didascalique et conversationnelle/narrative, s'interpénètrent, se confondent partiellement, ne se distinguant plus guère que par les polices de caractères : le dramaturge n'assure plus la fonction de régie sonore et scénique de la didascalie, devenue maintenant une contrescène dramatique.

Donc les didascalies apparaissent bien comme le prolongement de la ponctuation dans sa visée d'organiser et de préciser l'oralité, mais seulement dans les didascalies les plus 
courtes, des textes plus pédagogiques ou plus « classiques » réglés par un metteur en scène omniprésent. Toutefois les précisions vocales restent partielles, sans jamais atteindre le niveau de la partition musicale. Les didascalies en s'étoffant abandonnent peu ou prou cette fonction d'analyse clinique de la parole et de son cadre conversationnel pour assumer des fonctions plus narratives, poétiques et rythmiques : elles ébauchent des seconds plans qui réduisent l'épaisseur des personnages et le drame au bénéfice de mondes flottants de type poétique. Dans tous les cas, la didascalie nombreuse a tendance à devenir, dans le théâtre contemporain, une macroponctuation suspensive et rythmique (notre fonction 3).

\subsection{Didascalies et dialogues : de la cohabitation à la vie commune}

89 Notre point de départ trivial était la distinction habituelle de deux séquences, dialogue et didascalie, de deux régimes de langue, oral et écrit, de deux panoplies de ponctuants, et de façon implicite, de deux échelles d'unités discursives. L'unité discursive de base du dialogue devrait être la réplique, blanchie, entre phrase-période d'un côté, scène et pièce intégrale de l'autre, dans une distribution hiérarchique spécifique. Trouve-t-on une unité discursive didascalique correspondante, elle grisée par l'italique? Sans remettre en cause complètement le clivage séquentiel binaire, nous voudrions tenter de le nuancer par les pièces francophones contemporaines de notre corpus.

\subsubsection{Les ponctuants comparés des deux séquences}

90 La surprise vient de l'apparition, au sein des répliques orales, de ponctuants référés habituellement à la période écrite la plus classique :

91 Le deux-points est fréquent chez Mouawad (Journée de noces, p. 25) :

Mon père. Mais il n'est pas jeune et il ne porte jamais de chemise : ça lui irrite les aisselles.

On pourrait dire que le deux-points entraine brachylogie et parataxe typiques de l'oral. D'autres traits d'oralité se manifestent : la phrase nominale de lancement et de mise en valeur du focus: "Mon père", la place de l'adversatif entre le focus et sa reprise anaphorique par «il ». Pourtant nous avons bien ici une période interphrastique de cadence mineure. Le deux-points retrouve sa valeur d'apex de la période écrite classique, et de bascule intonative.

Le tiret et les parenthèses sont courants chez J.-L. Lagarce pour établir une véritable radiographie de période (La Fin du monde, p. 17) :

Et puis, et puisque vous n'aviez pas d'enfant, puisque vous n'avez pas d'enfantparce qu'il aurait été logique, nous le savons... ce que je voulais dire : mais puisque vous n'avez pas d'enfant et Antoine dit ça, tu dis ça, tu as dit ça, Antoine dit que vous n'en aurez pas - ce n'est pas décider de votre vie mais je crois qu'il n'a pas tort. Après un certain âge, sauf exception, on abandonne, on renonce - puisque vous n'avez pas de fils, c'est surtout cela, puisque vous n'aurez pas de fils, il était logique (logique, ce n'est pas un joli mot pour une chose à l'ordinaire heureuse et solennelle, le baptême des enfants, bon) il était logique, on me comprend, cela pourrait paraître juste des traditions, de l'histoire ancienne mais c'est aussi ainsi que nous vivons, il paraissait logique, nous nous sommes dit ça, que nous l'appelions Louis, comme votre père, donc, comme vous, de fait.

94 Ici le deux-points correspond à une relance secondaire ( - parce qu'il aurait été logique, nous le savons..- »). La ponctuation des ajouts parenthétiques, actualisant des 
paliers intonatifs, se fait par : 1) deux tirets qui prennent ici une valeur parenthétique, notamment parce qu'ils encadrent une incise ${ }^{4}$; 2) la double parenthèse, parenthétique en langue, avec son double signe symétrique ouvrant-fermant. On peut avancer que ces incises font deux opérations distinctes : celle avec parenthèses effectuant une boucle autonymique sur le mot « logique » et une répétition accentuante, celle avec tirets une boucle sur le dit mondain.

On aura noté l'usage du blanc interne, exceptionnel dans la prose périodique, écrite ou orale, qui, à une occurrence près, délimite des clauses entières soit uniques, soit regroupées en paquets homogènes de deux, trois ou quatre.

Ici, la ponctuation très sophistiquée correspond non seulement à la visée pragmatique de tenir l'interlocuteur en haleine, mais se rapproche sinon de la tabulation analytique du linguiste, du moins du tableau clinique du stylisticien, grâce notamment au jeu sur les blancs et les alinéas. La notion de "période ", voire de "paragraphe » utilisée par les linguistes de l'oral, comme A. Lacheret et B. Victorri, dit bien, mezzo voce, la filiation entre la période orale et la période écrite classique, et finalement le statut mixte de toute oralité transcrite. H. Deulofeu après C.Blanche-Benveniste (Deulofeu, 2014) s'était aussi posé la question de l'utilisation de la ponctuation pour retranscrire des enregistrements oraux : les ponctuants y ont l'inconvénient d'interpréter les énoncés oraux de façon toujours contestable, mais l'avantage de les actualiser et donc de leur (re)donner vie.

97 Le point-virgule, ponctuant par excellence de la cohésion syntaxique et sémantique à l'écrit, vite jugé par J. Dürrenmatt en voie de disparition (2015, p. 33 sqq.) y est employé également, dans les dialogues comme dans les didascalies. En fait, il est lié à la taille de la phrase; longue, elle a besoin de se structurer en parties, que sépare justement le point-virgule, devenu, plus que le deux-points de la période classique, le signe médian privilégié. Voyons une première occurrence de D.-G. Gabily, tirée d'une longue didascalie (1994, p. 41) :

La nuit tombe toujours aussi vite et avec elle vient la pluie - là-bas une bruine visible -, c'est encore le silence et une sorte de paix des (en) temps de guerre; c'est l'apaisement du monde comme s'il pouvait s'apaiser, le monde; ça ne ressemble à rien, sans doute, qu'à la paix du plateau, fallacieuse, pourtant bien réelle, inquiétante, si tout va bien.

La phrase périodique (ou période intraphrastique) comporte quatre moments et trois bornes ponctuationnelles de délimitation interne. Les trois derniers moments sont des reformulations de la première assertion : «c'est encore ; c'est ; ça ne ressemble à ». D.G. Gabily utilise deux points-virgules mais aucun entre le premier segment et la première reformulation. Pourtant la ponctuation entre le premier et le deuxième moment est très marquée, mais différentiellement: ici par un tiret, une virgule, et, dans notre théorie, par la dimension de l'apposition suspensive entre tirets, laquelle augmente la valeur de rupture du ponctuant double (Favriaud, 2011). Là pourrait être l'apex de la période avant l'apodose nombreuse de la cadence majeure.

La seconde, à la scène 18, est tirée d'une réplique tenue par «La Vieille» (1994, p. 43) :

[...] et je sus dès l'instant que je serais maudite par toutes les vieilles qui veillaient à

la continuation des cycles de nos morts; je le sus: que je me cacherais dans la montagne, apprenant de vous, bestioles, à picorer dans les coupes de pins [...]

Le point-virgule vient diviser une période d'onze lignes, dont la longue apodose se développe après l'anaphore de « je sus » et le deux-points, lequel marque à la fois la rupture énonciative et la relance. On ne saurait dire lequel des deux ponctuants, point- 
virgule ou tiret, prétend au rôle de sommet intonatif et rhétorique, mais il est bien possible que D.-G. Gabily retrouve ici l'échelle ascendante classique des intensités ponctuationnelles de la période classique écrite: du point-virgule au deux-points de l'apex.

Ici, nous n'avons pas démontré que la ponctuation des didascalies et celle des répliques étaient pour chaque auteur, encore moins dans tout le théatre contemporain, strictement égales, mais que la frontière entre les deux était souvent poreuse, que l'oralité des répliques ressemblait parfois à celle de la rhétorique écrite la plus classique quand on soulevait les oripeaux, et que, peut-être, les mêmes unités discursives étaient à l'œuvre, notamment la période.

\subsubsection{Les unités discursives comparées}

Nous avons avancé que la réplique était l'unité conversationnelle centrale, celle qui visuellement était la plus saillante, grâce à la ponctuation blanche interne et à la ponctuation grise de son lanceur. Y a-t-il une unité discursive de didascalie correspondante, et laquelle? Nous avons commencé à la circonscrire au moyen de la ponctuation grise de l'italique, en envisageant des didascalies courtes, mi-longues et longues, placées différemment dans les scènes, pouvant à elles seules faire scène chez D.-G. Gabily et A. Tarnagda.

Chacune de ces unités intercalées se compose de phrases et de périodes. Prenons l'exemple de D. Lemahieu qui fait un usage modeste des didascalies comme ici (Théâtre I) :

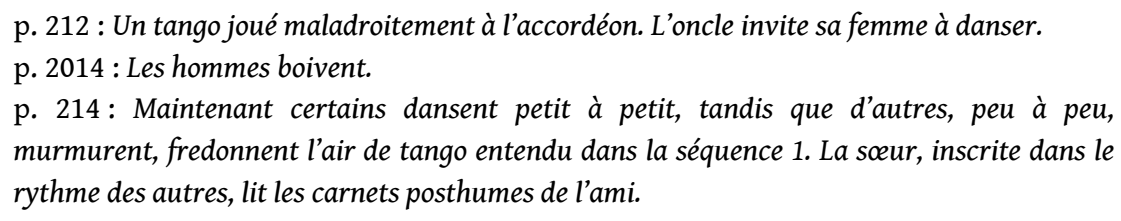

On continuera d'appeler phrase l'énoncé entamé par une majuscule et clos par un point ou un ponctuant final, qu'il soit à rection nominale ou verbale. Dès que la phrase s'allonge, la ponctuation médiane se multiplie, ici sous forme de virgules. Les première et troisième didascalies regroupent facilement leurs phrases en périodes interphrastiques. On comparera cette organisation ponctuo-discursive avec celle d'une réplique (ibid., p 216-217) :

MARILOU. - L'amour? L'amour? C'est fini l'amour. C'est réglé. C'est une conservation de l'espèce. Tout se ramène à ça. Je l'ai eu mon Pierrot. Mon enfant à moi. Pis quand ça n'existe plus y a plus rien. Tout simplement. Se reproduire ça sert à quelque chose à pas mourir c'est déjà quelque chose. J'ai été mal aimée mal aimée pas assez aussi surtout pas assez. [...]

On peut dire que les six premières phrases, dont deux nominales, font une période interphrastique, les deux suivantes une deuxième, etc. D. Lemahieu adopte dans les didascalies un dispositif ponctuationnel analytique, hiérarchisant et enchâssant les paliers, à l'aide de virgules: "dansent petit à petit, tandis que d'autres, peu à peu, fredonnent » (cit. supra, p. 214), et, dans les répliques, privilégie la phrase comme unité continue, interprétable par le lecteur et l'acteur.

Il semblerait que le couple phrase-période soit l'unité de base des répliques comme des didascalies, que les répliques et les didascalies soient les unités méso-textuelles intermédiaires. Une parenté relative, différente d'un auteur à l'autre, entre réplique et 
didascalie peut être avancée comme hypothèse. La didascalie quand elle occupe toute une scène, quand elle est introduite par un nom de locuteur, comme chez D.-G. Gabily (voir supra), nous aide à penser, toute proportion gardée, que la didascalie puisse être elle-même, asymptotiquement, une réplique - mais d'un autre type.

Jusqu'ici nous avons insisté sur les ressemblances de ponctuation et de construction discursive ; il serait temps de clore sur quelques différences habituellement reconnues.

Au plan de la ponctuation, on s'arrêtera sur les points de suspension, extrêmement rares dans les didascalies, mais fréquentes dans les répliques. J.-M. Dalpé les loge soit en fin de phrase, soit en cours (Le Chien, p. 35) :

On dit: ça se peut pas, pas tout ce temps-là, pas juste des comptes!... Ben oui, des comptes pis c'est toute ! « C'est ben cher l'hydro c'mois-citte... Oublie pas de toute noter dans le livret en commun d'la caisse... Pourquoi t'achètes ça des «brocolis »? Tu payes plus, juste juste parce que ça l'a un nom italien... [...] J'me suis fâchée un soir que j'avais pris un verre de trop... là, y m'a frappée avant de partir. Un hiver en attend pas un autre, pis les comptes ont été payés pendant vingt ans à tous les débuts de mois. J'ai pris mon trou. Y m'a laissé le prendre. Pis à c'heure, ben que le'Yable l'emporte... que l'Yable l'emporte, lui pis ses comptes... que l'Yable l'emporte!

109 Quand les points de suspension sont suivis d'une majuscule, les différentes phrases se regroupent en périodes interphrastiques très animées (alinéa 1) car cette double ponctuation, noire et grise, crée un événement intonatif et rythmique, un contreaccent fort de début de phrase. Quand suivis d'une minuscule (alinéa 2 de la citation), les énoncés s'enchainent en une phrase périodique unique, à la fois continue et discontinue. C'est une nuance qui peut avoir son effet de sens, surtout quand les deux types de périodes cohabitent. Mais les points de suspension, qui suspendent le dire plus que le dit, sont des signes au sémantisme flou, qui laissent une marge de manœuvre importante à l'acteur et au metteur en scène, et qui créent, pour le lecteur, plus un rythme visuel et une atmosphère sémantique qu'un sens précis. Ici, les points de suspension ajouteraient une valeur métacognitive et métalinguistique : la phrase et la période sont en train de se faire, avec des limites flottantes, par ajouts. Ils témoigneraient du langage sortant de sa gangue chaude, tandis que la phrase ordinairement ponctuée, modèle unique dans la didascalie, semble posée de façon abstraite. Le théâtre mettrait ainsi au coude à coude moins les deux régimes traditionnels de langage, écrit et oral, que deux états processuels du langage, un langage d'amont et un langage d'aval, séparés dans le temps, dans la conscience et dans le rapport à la norme.

110 Ce travail de la phrase et de la période en cours d'élaboration peut se marquer par l'épéxégèse intensifiée (Gabily, 1994, p. 13) :

Mon nom ne qualifie rien. A peine si je m'en souviens moi-même. Quand je l'aurai totalement oublié, viendra le. Quoi. Rien. J'ai peur aussi de cet oubli, de ce. Quoi. Rien. J'ai peur.

Ces neuf phrases peuvent se regrouper en trois périodes interphrastiques, dont la première recouvrirait les deux premières phrases, la deuxième, les trois suivantes, etc. Ici, les ruptures-ajouts autour du point scindent le syntagme nominal en un lieu dit insécable, après le déterminant («le», «ce»). Les ajouts de phrases, réitérés («Quoi. Rien. »), marquent à la fois la suspension du discours, l'interrogation sur la modalité et le sémantisme lexical : un « Quoi » sans point d'interrogation, un mot « Rien » qui peut 
être pris comme pronom indéfini ou comme nom, peut-être même nom propre, majusculé.

\section{Conclusion?}

Donc, dans le théâtre contemporain, privé de versification, de vers réguliers et d'enjambements, rejets et contre-rejets, la ponctuation c'est la vie d'un oral trempé dans l'écrit, à condition de ne pas limiter la ponctuation à la noire, ni même au plurisystème ponctuationnel.

$\mathrm{Au}$ plan discursif, le couple phrase-période fonctionne assez semblablement dans la didascalie et dans la réplique conversationnelle, mais la phrase n'y joue pas tout à fait le même rôle. Dans le conversationnel, la phrase anime la période, assure l'agôn, la tension et l'agitation diégétique, rythmique et cognitive. On ne s'étonnera pas que ce soit la phrase qui porte la ponctuation suspensive: points d'interrogation et d'exclamation, mais aussi points de suspension, qui laissent transparaitre, comme l'épéxégèse, le processus vivant, émotionnel, parfois hésitant et indiscipliné du discours (voir Favriaud \& Mignon, 2012). La différence linguistique entre les deux séquences tiendrait au moins autant à l'aspectualité constitutive de la phrase comme processus - inchoatif, en fusion, expérimentation, jaillissement dans les répliques plus achevé et abstrait dans les didascalies - qu'à une différence d'unités discursives ou de régime écrit/oral.

114 Les didascalies se sont imposées de plus en plus à la fin du $\mathrm{XX}^{\mathrm{e}}$ siècle, comme séquence majeure de l'écriture dramatique, parfois à égalité avec les répliques conversationnelles, au fur et à mesure que l'intrigue et ses personnages s'effritaient. Elles jouent maintenant un double rôle : celui ancien d'assistant de régie scénique et vocale, en complément de la ponctuation des répliques (nos fonctions 1 et 2) ; et celui, plus nouveau - ou très ancien - de macro-ponctuation rythmique, si l'on pense qu'elles assument certaines des fonctions du chœur antique (notre fonction 3). Les didascalies apparaissent bien elles-mêmes comme des macro-ponctuants dramaturgiques d'un autre type, réglés par leur taille, qui, à la lecture au moins, suspendent, parfois longuement, l'intrigue, l'annotent pleine page, la font dériver jusqu'à des contrescènes, avec éventuellement des répliques. Véritable défi scénique et lieu de création.

Plus encore que la multiplication des points d'exclamation, d'interrogation et de suspension dans les répliques, différente selon les auteurs, les didascalies apportent à la pièce une nouvelle oralité, au sens du rythme meschonnicien cette fois, c'est-à-dire un contre-chant poétique, infiniment modelable et interprétable. Car, comme tout ponctuant, elles sont souvent moins prescriptives que délibératives, donc potentiellement créatrices.

\section{BIBLIOGRAPHIE}




\section{Corpus des pièces de théâtre}

Dalpé. J.-M. (2003). Le Chien. Sud Bury : Éd. Prise de parole.

Gabily, D.-G. (1994). Chimère et autres bestioles. Arles : Éd. Actes Sud.

Kane, S. (1999). L'Amour de Phèdre. Paris : Éd. de L'Arche.

Koltès, B.-M. (1985). Quai Ouest. Paris : Éd. de Minuit.

Lagarce, J.-L. (1999). Juste la fin du monde. Besançon : Éd. Les Solitaires intempestifs.

Lemahieu, D. (1997). Théâtre I. Pézenas : Domens.

Mouawad, W. (2011). Journée de noces chez les Cromagnons. Arles : Actes Sud.

Novarina, V. (1998). L'Opérette imaginaire. Paris : P.O.L.

Novarina, V. (1984). Le Drame de la vie. Paris : Gallimard.

Tarnagda, A. (2019). Musika. Carnières-Morlanwelz : Lansman.

Tremblay, M. (1984-2007). Albertine. En cinq temps. Arles : Actes Sud.

Zambon, C. (1998). Sissi pieds-jaunes. Paris : Éd. L'École des loisirs.

\section{Études}

Anis, J. (2004). « Les linguistes français et la ponctuation ». L'Information grammaticale, 102, p. 5-10. Béguelin, M.-J. (2002). « Clause. période ou autre ? La phrase graphique et la question des niveaux d'analyse ». in : Charolles, M., Le Goffic, P. \& Morel, M.-A. (éds). Y a-t-il une syntaxe au-delà de la phrase?. Nancy : Presses universitaires de Nancy, p. 85-108.

Benveniste, É. (1966). Problèmes de linguistique générale 1. Paris : Gallimard.

Benveniste, É. (1974). Problèmes de linguistique générale 2. Paris : Gallimard.

Berrendonner, A. (2002). « Les deux syntaxes ». in : Charolles, M., Le Goffic, P. \& Morel, M.-A. (éds). Y a-t-il une syntaxe au-delà de la phrase ?. Nancy : Presses universitaires de Nancy, p. 23-36. Berrendonner, A. (2021). « La notion de phrase ». Encyclopédie grammaticale du français. En ligne : http://encyclogram.fr.

Bottineau, D. (2013). « Pour une approche énactive de la parole dans les langues ». Langages, 192, p. 11-28.

Charolles, M. (1988). « Les plans d'organisation textuelle : périodes, chaînes, portées et séquences ». Pratiques, 57, p. 3-13.

Damourette, J. (1939). Traité moderne de ponctuation. Paris : Larousse.

Dessons, G. \& Meschonnic, H. (1998). Traité du rythme. Paris : Dunod.

Deulofeu, H.-J. (2011). « Peut-on établir un système de ponctuation des transcriptions de textes oraux linguistiquement fondé ». Langue française, 172, p. 115-131.

Dürrenmatt, J. (2015). La Ponctuation en français. Paris : Éd. Ophrys.

Favriaud, M. (2010). «Le Traité moderne de ponctuation de Jacques Damourette : une théorie inachevée du sujet vocal dans le texte ». in : De la grammaire à l'inconscient - dans les traces de 
Damourette et Pichon. Arrivé, M., Muni Toke. V. \& Normand. C. (éds). Limoges : Lambert-Lucas. p. 37-44.

Favriaud, M. (2011). «Plurisystème ponctuationnel. Dimension, intensité des signes et architecturation du texte poétique » Langue française, 172, p. 83-98.

Favriaud, M. \& Mignon, F. (2012). «La ponctuation dans Juste la fin du monde de Jean-Luc Lagarce : une mise en pièce du discours ». in : Doquet, C. \& Richard, E. (éds). Continuité, discontinuité, reprise : des représentations de l'oral chez Jean Luc Lagarce. Louvain-la Neuve : Éd. Academia Bruylant, p. 65-86.

Favriaud, M. (2014). Le plurisystème ponctuationnel français à l'épreuve de la poésie contemporaine. Limoges : Lambert-Lucas.

Kolinski, R. et al. (2014). «L'influence de l'apprentissage du langage écrit sur les aires du langage ». Revue de psychologie, 6, p. 173-181.

Lacheret, A. \& Victorri, B. (2002). « La période intonative comme unité d'analyse pour le français parlé » Verbum, t. XXIV, 1-2. Nancy : Presses universitaires de Nancy, p. 55-72.

Petitjean, A. (2012). Études linguistiques des didascalies. Limoges : Lambert-Lucas.

Wilmet, M. (1997). Grammaire critique du français. Louvain-la-Neuve : Duculot.

\section{NOTES}

1. Nous appelons poémateur l'instance énonciatrice du poème, en démarquage du narrateur, instance du récit.

2. J. Damourette (1939) fait une place dans son Traité moderne de ponctuation aux accents orthographiques (p. 134). On peut se demander si le « â » long ne déplace pas l'accent sur la première syllabe.

3. Nous entendons ici par oralité le champ de l'oral parlé, même si le sens meschonnicien de rythme des discours écrits et oraux pourrait in fine être rejoint.

4. Nous récusons la notion de tiret double, qui serait en langue un synonyme des parenthèses et un homonyme du tiret unique (Favriaud, 2014).

\section{RÉSUMÉS}

Cet article traite de la ponctuation dans le théatre contemporain de langue française et de sa relation à l'oral dans les didascalies. Même la ponctuation étendue (le plurisystème ponctuationnel, Favriaud, 2014) est incapable de rendre compte de tous les traits d'oralité, ce que les didascalies pourraient aider à faire. Mais la différence entre séquences conversationnelle et didascalique s'amenuise aujourd'hui (Petitjean, 2012) ; les deux utilisent les mêmes unités discursives, la même ponctuation (à l'exception de la ponctuation modale). Notre hypothèse est que les didascalies sont à la fois un complément de ponctuation au service des répliques, et une seconde scène (semblable au choeur antique) faisant elle-même ponctuation et rythme. 
This article deals with punctuation in contemporary French speaking theater and its relation with orality mainly through didascalia. Even extended punctuation (Favriaud's black, blank, graphic one, 2014) is unable to figure out all the features of orality; so stage directions may do part of the job. Actually the differences between replica and didascalia are diminishing nowadays (Petitjean, 2012); both use the same discourse units, and almost the same punctuation grades except modal points which are specific of conversation. Our hypothesis is that didascalia are partly an oral and physical complement of the replica punctuation, partly a second stage (similar to greek chorus), providing punctuation and rythm of a new kind.

\section{INDEX}

Keywords : extended punctuation, contemporary theater, orality, didascalia, sentence, period. Mots-clés : ponctuation étendue, théâtre contemporain, oralité, didascalie, phrase, période

\section{AUTEUR}

\section{MICHEL FAVRIAUD}

Université de Toulouse - Jean Jaurès, F-31058 Toulouse, France 